\title{
HEAVY ION MEASUREMENT ON LDEF
}

\section{N92-23308}

\author{
R. Beaujean, D. Jonathal and W. Enge \\ Institut für Reine und Angewandte Kernphysik \\ Christian-Albrechts-Universität zu Kiel, D-2300 Kiel, FRG \\ Phone: (0) $431 / 880-2544$, Fax: (0) $431 / 85660$
}

\section{SUMMARY}

Stacks of CR-39 and Kodak CN track detectors were exposed on different locations on the NASA satellite LDEF. The preliminary analysis yielded heavy ion tracks on a background of low energy secondaries from proton interactions. The detected heavy ions with energies $<50 \mathrm{MeV} /$ Nuc show a steep energy spectrum and a spatial confinement close to the mirror plane in the South Atlantic Anomaly. We interpret this as evidence for a radiation belt origin.

\section{INTRODUCTION}

The Kiel LDEF experiment M0002, mounted on experiment tray E6, was designed to measure the heavy ion environment by means of CR-39 plastic solid state track detectors. The detector stack with a size of $40 * 34 * 4.5 \mathrm{~cm}^{3}$ was exposed in vacuum covered by thermal protection foils with a total thickness of approximately $14 \mathrm{mg} / \mathrm{cm}^{2}$.

Two additional stacks, each with a size of $40 \mathrm{~mm}$ depth and $95 \mathrm{~mm}$ diameter, were integrated in subunits of the Biostack Experiment A0015 on trays C2 and G2. They consist of CR-39 and Kodak cellulose nitrate foils sealed in aluminium containers under $10 \mathrm{mg} / \mathrm{cm}^{2}$ Kapton shielding and support the analysis of the spatial particle distribution.

All stacks were recovered in excellent physical condition with no damage on the thermal protection foils. After etching, tracks of heavy ions can be easily detected in both detector types on a background of small etch pits, which were probably produced by secondaries from proton interactions (Fig. 1).

\section{PRELIMINARY RESULTS}

In the first step of our analysis the appropriate etching condition had to be established, as the total number of tracks and the detector response were undefined. At the time being we are not able to present a calibration for our detector material and we must be aware of a potential temporal change of the detector response during the mission. Any particle flux measurement strongly depends on this detector response. 
Table 1 gives numbers of stopping tracks in CR-39 foils at position E6. From the estimated sensitivity of our CR-39 we conclude that particles with $Z>8$ are included. Similar decrease of stopping particle numbers with increasing depth was detected in Kodak $\mathrm{CN}$ on trays C2 and G2 but at a 3-5 times higher fluence level. If our Kodak $\mathrm{CN}$ is more sensitive then CR-39, particles with $\mathrm{Z} \geq 6$ may be registered in Kodak $\mathrm{CN}$. The work on the calibration of the detector response is in progress.

As the LDEF orientation with respect to the magnetic field lines within the South Atlantic Anomaly (SAA) is expected to be constant during the mission, we measured the azimuth angle distribution on the detector foils (Figs. 2 and 3) for particles entering the topmost CR-39 foils at trays E6 and C2.

\section{DISCUSSION}

The azimuth angle distribution of Fig. 2 shows characteristics of a cylindrical symmetry. This is supported by the distribution of Fig. 3: On tray C2 the detected particles entered from the earth direction. Including the dip angle measurements we can deduce that all detected arrival directions are close to a plane perpendicular to the magnetic field line of $-20^{\circ}$ declination and $-40^{\circ}$ inclination (location $34^{\circ} \mathrm{W}$ and $27^{\circ} \mathrm{S}$ within the SAA).

From Table 1 we deduce a steep energy spectrum similar to the trapped proton spectrum. Together with the spatial distribution we take this as an evidence for the detection of heavy ions from a radiation belt population. Similar results were reported from Skylab by Chan and Price (ref. 1) and from Spacelab 1 by Beaujean et al. (ref. 2). At the present stage of our analysis neither flux values nor charge spectra can be given.

In addition to particles with energies < $50 \mathrm{MeV} / \mathrm{Nuc}$ we detected particles which penetrate several sheets with almost constant energy loss. The arrival directions and the energies ( $100 \mathrm{MeV} / \mathrm{Nuc}$ ) are not yet determined. This particle flux is in agreement with predicted LET spectra for LDEF including cosmic rays from $\mathrm{H}$ to $\mathrm{U}$. They are mainly produced by $\mathrm{Fe}$-particles and their fragments after traversing LDEF material.

This work was financially supported by the "Bundesministerium für Forschung und Technologie" under grant $01 \mathrm{QV} 297$ and 50 OS 9001. We like to thank the DLR-Flugmedizin (Köln-Porz) for the integration of our detector foils in experiment A0015.

\section{REFERENCES}

1. J.H. Chan and P.B. Price: Composition and Energy Spectra of Heavy Nuclei of Unknown Origin Detected on Skylab; Phys. Rev. Lett. Vol. 35, \#8, 539, 1975.

2. R. Beaujean, K. Oschlies and W. Enge: Components of the Low Energy Cosmic Ray Oxygen Flux Measured on Spacelab 1, SH-21, 12th ECRS, Nottingham, 1990. 


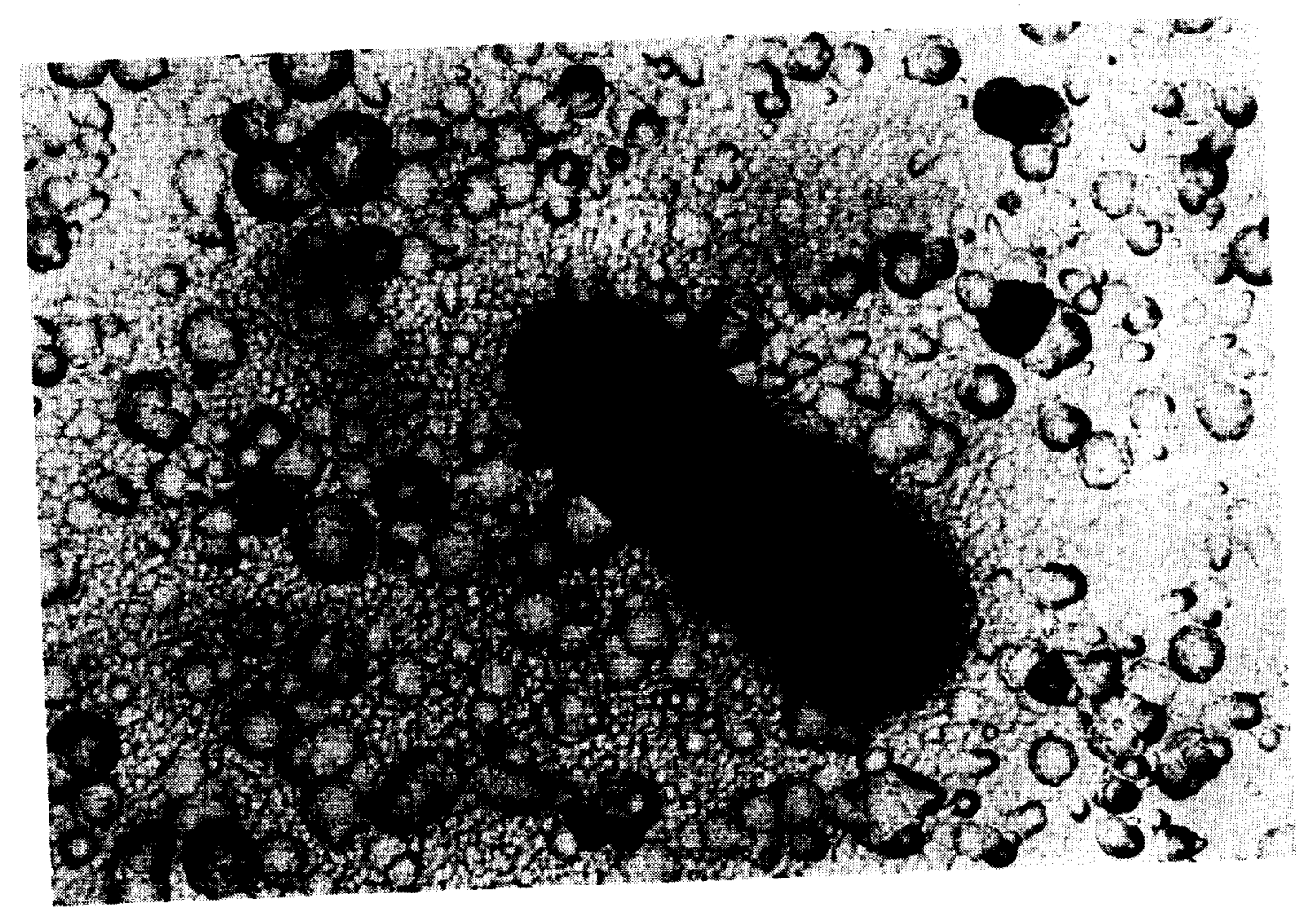

Fig. 1: Track images in CR-39 of M0002 (E6)

Table 1:

Number of stopping particles in the topmost foils of M0002 (E6).

The range intervalls, the mean range $R$ and the corresponding energy intervalls and the mean energy $E$ are calculated for a $45^{\circ}$ incident angle.
stopping
$\overline{\mathbf{R}}$
tracks $/ \mathrm{cm}^{2} \quad[\mu \mathrm{m}]$
$\overline{\mathbf{E}}[\mathrm{MeV} / \mathrm{NuC}]$
$\mathrm{O}^{16} \quad \mathrm{Fe}^{56}$
Foil $1 \quad 3.0 \pm 0.35^{1)} \quad 295\left(\begin{array}{l}154- \\ 546\end{array}\right) \quad 10.8\left(\begin{array}{c}7.3- \\ 15.5\end{array}\right) \quad 17.7\left(\begin{array}{l}11.2- \\ 26.3\end{array}\right)$
Foil $2 \quad 0.3 \pm 0.05^{2)} \quad 710\left(\begin{array}{c}546- \\ 1050\end{array}\right) \quad 18.0\left(\begin{array}{c}15.5- \\ 22.5\end{array}\right) \quad 31.0\left(\begin{array}{l}26.3- \\ 39.5\end{array}\right)$
Foil $3 \quad 0.1 \pm 0.03^{3)} 1210\left(\begin{array}{l}1050^{-} \\ 1554\end{array}\right) 24.5\left(\begin{array}{l}22.5- \\ 28.0\end{array}\right) \quad 43.0\left(\begin{array}{l}39.5- \\ 49.0\end{array}\right)$

1) 75 stopping tracks $\left./ \begin{array}{r}25 \mathrm{~cm}^{2} ; \\ 100 \mathrm{~cm}^{2} ;\end{array}\right\}$ Poissonian error; scanning efficiency not included

3) 10 stopping tracks $/ 100 \mathrm{~cm}^{2} ;$ 


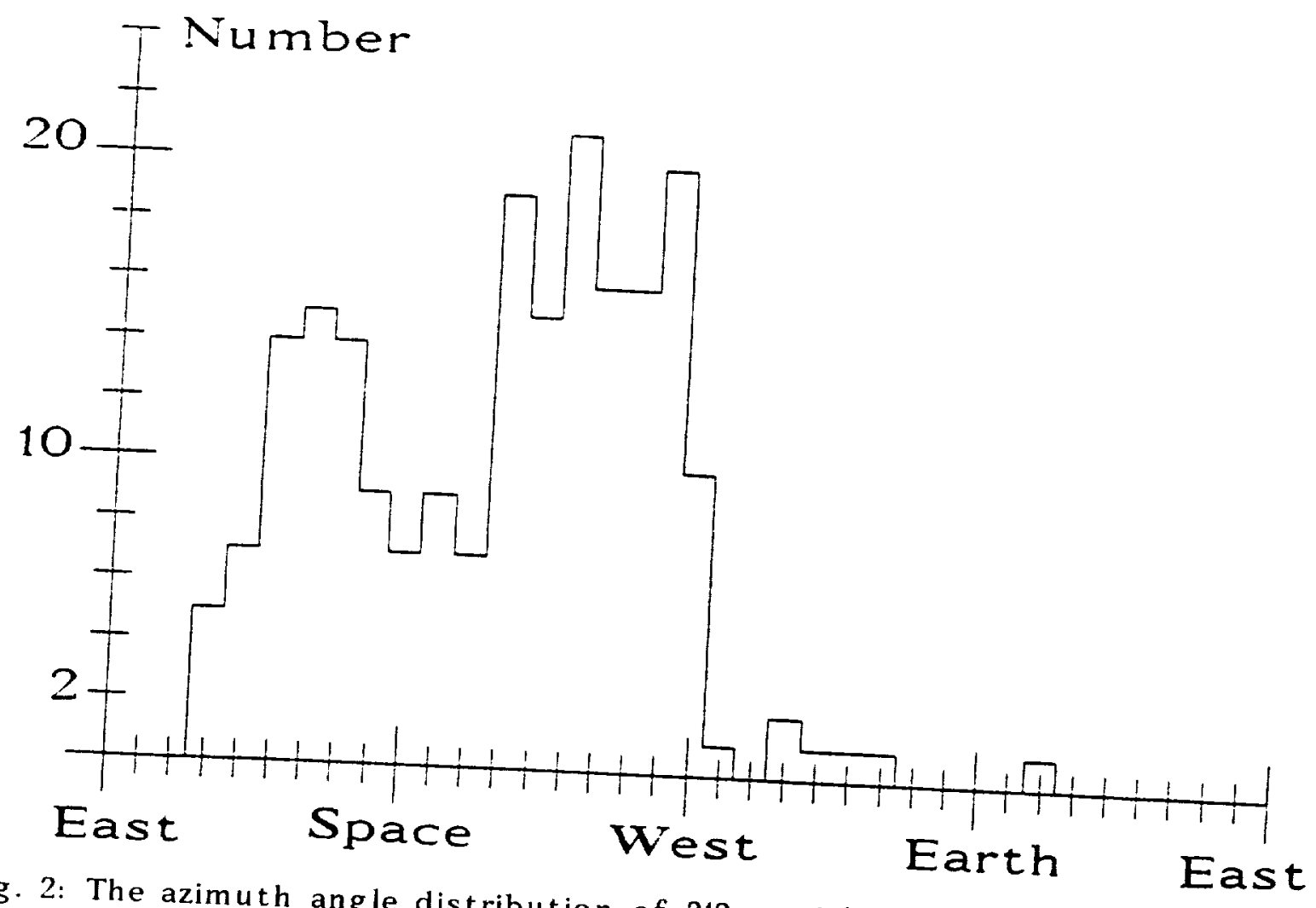

Fig. 2: The azimuth angle distribution of 212 particles entering foil 1 of M0002 (E6)

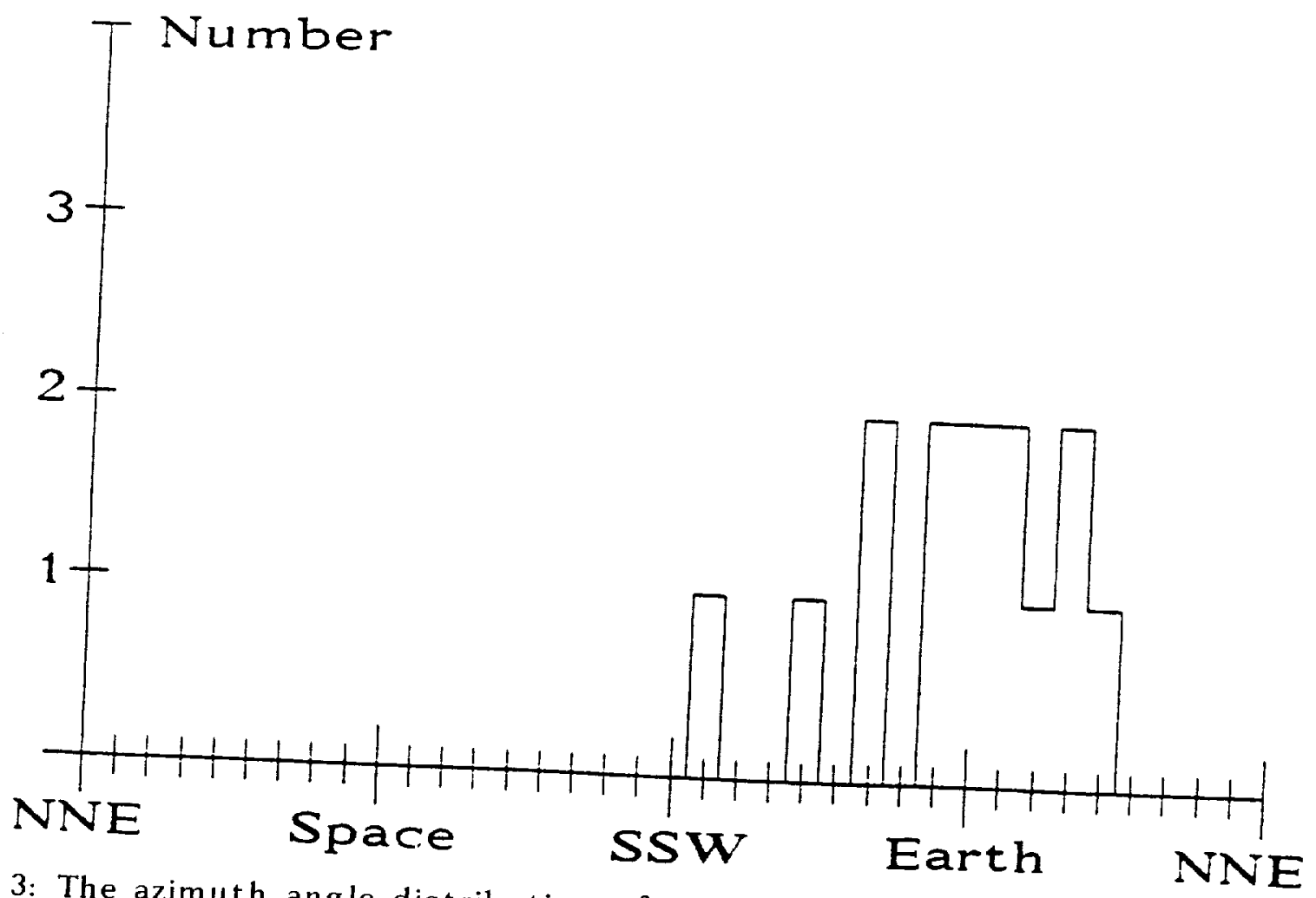

Fig. 3: The azimuth angle distribution of 14 particles entering A0015 (C2) 


\section{SPACE ENVIRONMENTS METEOROID AND DEBRIS}

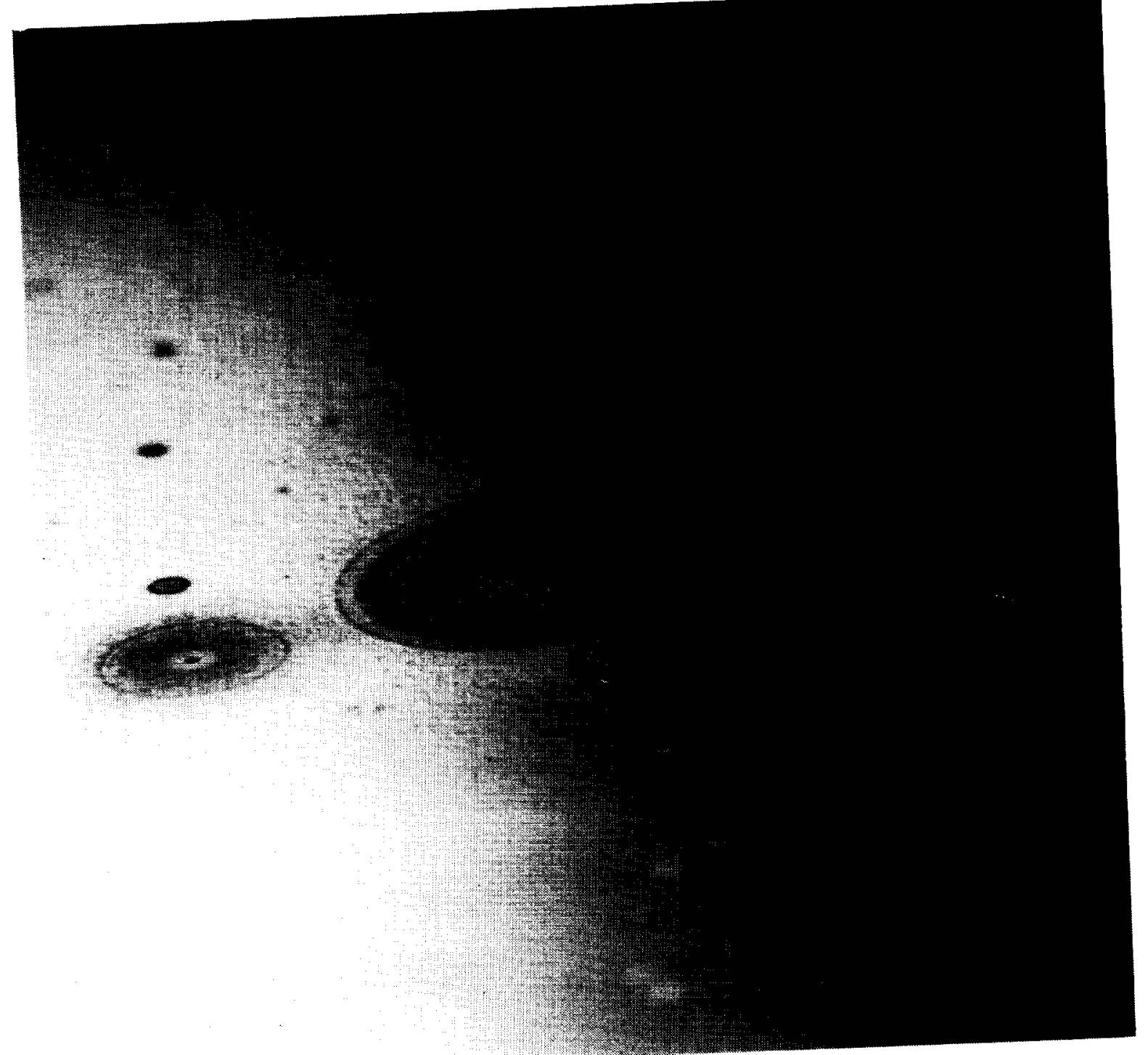


